1993 to $9.0 \%(123 / 1373)$ in 1998 , an adjusted average decrease of $9 \%$ a year $(95 \%$ confidence interval decrease $1 \%$ to $16 \%)$. When specimens from men known to be HIV-1 positive were excluded there was no adjusted trend in prevalence, which was 4.9\% $(64 / 1314)$ in 1998. The adjusted decline in prevalence of HIV-1 among homosexual and bisexual men presenting with non-acute sexually transmitted infections was significant: $17 \%$ a year (12\% to $21 \%)$ overall and $11 \%$ a year $(7 \%$ to $16 \%)$ when men known to be HIV-1 positive were excluded.

Outside London, the observed prevalence of HIV-1 among homosexual and bisexual men was lower (figure), and the adjusted decrease in prevalence overall was significant only in men presenting with non-acute sexually transmitted infections (average decrease of $11 \%$ a year ( $4 \%$ to $18 \%)$ ).

The proportion of all attenders with an acute sexually transmitted infection increased over time in London, but not outside London. This trend was less apparent when attenders known to be HIV-1 positive were excluded.

\section{Comment}

In 1993-8 the prevalence of undiagnosed HIV-1 infection in homosexual and bisexual men presenting with acute sexually transmitted infection did not fall, which indicates a high level of continuing transmission. The smaller increase in this group of men in London is consistent with a progressive drawing away of men infected with HIV from participating clinics or a reduction in syphilis testing of HIV infected attenders without an acute infection.
The difference in trends between men with and without acute sexually transmitted infections may be partly because of repeat sampling of a core group of HIV-1 infected men with repeated new infections. If so, it will represent a marker of significant risk for HIV-1 transmission in the population. These men probably have more partners and engage in riskier sex than those without an acute sexually transmitted infection. ${ }^{4}$ The facilitatory effect of many acute infections on HIV-1 transmission ${ }^{5}$ may also have contributed to the higher prevalence. Health promotion directed at this group of men should be intensified.

Contributors: MAC, ONG, and DM conceived and designed the study. PAR was responsible for the statistical analysis in collaboration with CAMcG and LFJ. CAMcG and LFJ coordinated the study. CAMcG interpreted the data together with MAC and PAR. MAC, CAMcG, and ONG prepared the paper in consultation with the other authors. MAC is the guarantor for the paper. Funding: Department of Health.

Competing interests: None declared.

1 Unlinked Anonymous HIV Surveys Steering Group. Prevalence of HIV in the United Kingdom, data to end 1998. London: Department of Health, 1999.

2 Nardone A, Rogers P, Joyce C, Nicoll A, Mercey D. The interpretation of trends in HIV seroprevalence amongst homo/bisexual men attending a GUM clinic in central London. MRC AIDS Research Workshop Abstract Book 1995;4-5:29.

3 Simms I, Rogers P, Catchpole M, McGarrigle CA, Nicoll A on behalf of the collaborative group. Trends in undiagnosed HIV-1 infection among attenders at genitourinary medicine clinics: 1990-6. Sex Transm Inf 1999;75:332-6.

4 Catchpole M, Connor N, Brady A, Kinghorn G, Mercey D, Band B, et al. Behavioural and demographic characteristics of attenders at two genitourinary medicine clinics in England. Genitourin Med 1997:73:457-61.

5 Grosskurth H, Mosha F, Todd J, Mwijarubi E, Klokke A, Senkoro K, et al. Impact of improved treatment of sexually transmitted diseases on HIV infection in rural Tanzania: randomised controlled trial. Lancet 1995;346:530-6.

(Accepted 5 July 2000)

\title{
Body mass and probability of pregnancy during assisted reproduction treatment: retrospective study
}

\author{
J X Wang, M Davies, R J Norman
}

Department of

Obstetrics and

Gynaecology,

University of

Adelaide, Queen

Elizabeth Hospital,

Woodville, SA 5011 ,

Australia

J X Wang

senior research officer

M Davies

senior research fellow

R J Norman

professor of

reproductive medicine

Correspondence to:

J X Wang

jwang@medicine.

adelaide.edu.au

BMJ 2000;321:1320-1
Being underweight or overweight has an adverse effect on reproduction. ${ }^{12}$ Overweight women have a higher incidence of menstrual dysfunction and anovulation, possibly because of altered secretion of pulsatile gonadotropin releasing hormone, sex hormone binding globulin, ovarian and adrenal androgen, and luteinising hormone and also because of altered insulin resistance. The prevalence of obesity in infertile women is high, but there is no conclusive evidence that extremes of weight are associated with a low rate of pregnancy in women receiving assisted reproduction treatment. This study examined whether body mass index (weight $(\mathrm{kg}) /\left(\right.$ height $\left.\left.(\mathrm{m})^{2}\right)\right)$ is associated with reduced fecundity (the probability of achieving at least one pregnancy during treatment) in women receiving assisted reproduction treatment.

\section{Participants, methods, and results}

The participants were 3586 women who received assisted reproduction treatment between 1987 and 1998 in a tertiary medical unit in Adelaide, South Australia.
Treatments included in vitro fertilisation $(n=1972)$, intracytoplasmic sperm injection $(n=1040)$, and gamete intrafallopian transfer $(n=574)$. Patients underwent 8822 embryo transfer cycles. The overall implantation rate was $12.0 \%$, and the clinical pregnancy rate was $24.1 \%$ in the study population. Causes of infertility included tubal blockage (34\%), semen defects (35\%), unexplained infertility (16\%), and endometriosis $(9 \%)$. Age of participants, treatment modalities, location of the treatment, number of embryos transferred, number of cycles of embryo transfer, and number of oocytes recovered were analysed to eliminate possible confounding effects. Polycystic ovarian syndrome was diagnosed, using normal criteria, in 25\% (881/3586) of the women. ${ }^{3}$

Participants were stratified into five groups according to body mass index: "underweight" $(<20)$, "moderate" (20.0-24.9), "overweight" (25.0-29.9), "obese" (30.0-34.9), and "very obese" ( $\geqslant 35)$. Fecundity was defined as the probability of achieving at least one pregnancy throughout the treatment. Pregnancy was determined by ultrasonography of the embryonic sac (or sacs) in the womb at 4-6 weeks after embryo transfer. 
Influence of body mass on probability of pregnancy during assisted reproduction treatment. Figures are values (SD) unless stated otherwise

\begin{tabular}{|c|c|c|c|c|c|c|c|}
\hline Category & $\begin{array}{l}\text { Body mass } \\
\text { index }\end{array}$ & $\begin{array}{c}\text { No of women } \\
(n=3586)\end{array}$ & Age (years)* & $\begin{array}{l}\text { No of embryos } \\
\text { transferred }\end{array}$ & No of cycles & $\begin{array}{c}\% \text { achieving at } \\
\text { least one } \\
\text { pregnancy } \dagger\end{array}$ & Odds ratioł \\
\hline Underweight & $<20$ & 441 & $31.6(4.5)$ & $2.4(0.8)$ & $2.3(1.5)$ & 45 & 0.81 (0.65 to 1.01$)$ \\
\hline Moderate & $20-24.9$ & 1910 & $32.9(4.7)$ & $2.4(0.7)$ & $2.3(1.7)$ & 48 & 1 \\
\hline Overweight & $25-29.9$ & 814 & $33.0(4.8)$ & $2.4(0.8)$ & $2.2(1.5)$ & 42 & 0.81 (0.68 to 0.97$)$ \\
\hline Obese & $30-34.9$ & 304 & $32.8(4.7)$ & $2.4(0.7)$ & $2.1(1.4)$ & 40 & 0.73 (0.57 to 0.95$)$ \\
\hline Very obese & $\geqslant 35$ & 117 & 32.7 (5.1) & $2.4(0.7)$ & $2.0(1.3)$ & 30 & 0.50 (0.32 to 0.77$)$ \\
\hline
\end{tabular}

The clinical protocols have been described elsewhere. ${ }^{4}$ We compared the groups by using analysis of variance and a $\chi^{2}$ test. We assessed the effect of body mass index, controlling for the confounding factors, by logistic regression.

The number of treatment cycles and embryos transferred per cycle did not differ among the groups, but age varied significantly but unsystematically (table). There was a significant linear reduction in fecundity from the moderate group to the very obese group $(\mathrm{P}<0.001)$. The fecundity of the moderate group was almost $60 \%$ higher than that of the very obese group, and the fecundity of the underweight group was also significantly lower than that of the moderate group $(\mathrm{P}<0.05)$, indicating an "inverted U" relation between body mass index and fecundity.

Logistic regression analysis confirmed the independent effect of body mass on fecundity. When the significant effects of maternal age, number of embryos transferred, number of cycles received, treatment type, and cause of infertility were controlled for, the pregnancy rate among very obese women was half that of the moderate group. Polycystic ovarian syndrome had an independent effect on fecundity.

\section{Commentary}

A body mass index that was either high or low was associated with reduced probability of achieving pregnancy in women receiving assisted reproduction treatment. Mechanisms through which body mass affects reproduction that have been cited include menstrual disturbance and anovulation, ${ }^{5}$ but these problems can be overcome through assisted reproduction treatment. There is no evidence that body mass affects the quality of the embryo and therefore the pregnancy rate. We propose that other mechanisms, such as altered receptivity of the uterus after transfer of embryos or oocytes, possibly because of disturbed endometrial function, may cause reduced fecundity.

We thank the staff of the Reproductive Medicine Unit in Adelaide for their contribution.

Contributors: JXW and RJN conceived and designed the study. JXW analysed and interpreted the data and wrote the paper. MD assisted in the analysis and interpretation of data, and $\mathrm{MD}$ and $\mathrm{RJN}$ revised the paper.

Funding: No additional funding.

Competing interests: None declared.

1 Correa H, Jacoby J. Nutrition and fertility: some iconoclastic results. Am J Clin Nutr 1978;31:1431-6.

2 Frisch RE. Body weight and reproduction [letter]. Science 1989;246:432.

3 Norman RJ, Masters SC, Hague W, Beng C, Pannall P, Wang JX. Metabolic approaches to the subclassification of polycystic ovary syndrome. Fertil Steril 1995;63:329-35.

4 Norman RJ, Warnes GM, Wang X, Kirby CA, Matthews CD. Differential effects of gonadotrophin-releasing hormone agonists administered as desensitizing or flare protocols on hormonal function in the luteal phase of hyperstimulated cycles. Hum Reprod 1991;6:206-13.

5 Lake JK, Power C, Cole TJ. Women's reproductive health: the role of body mass index in early and adult life. Int J Obes Relat Metab Disord 1997;21:432-8.

(Accepted 2 July 2000)

\section{A memorable patient}

\section{Sometimes life isn't fair}

I was working as a locum house officer during my vocational training in a local teaching hospital. The senior house officer and I had split the work between us, he to the accident and emergency department and I had gone to the general practitioner admission unit. As always the flow of patients was relentless, but as the day wore on I became familiar with some of the friendly faces of the patients on the unit. Some were my patients, others were awaiting transfer.

After several hours of work, a charming elderly woman caught my gaze. "I've been watching you all day, rushing about. Have you had your lunch? I'm worried about you." I was quite taken aback; after all she was the one who had been in hospital for a few days. She was the one who needed looking after. The day plodded on and each time I walked past her she would ask how I was feeling, making sure that I'd taken a break and had something to drink.

About 15 hours had passed without any break and I was writing up notes when there was a little commotion among the nurses, something about a patient fainting. The second-on house officer was called and he asked me for my advice about the patient.

The patient hadn't fainted; she'd had a stroke-and this was the woman who had been so kind to me. I spoke to her: "Do you remember who I am?" She stared at me blankly and asymmetrically, her dense rightsided weakness obvious to all.

After I gave my advice I returned to my notes and did something I hadn't done for a long time. I cried. Sometimes life just isn't fair.

William Murdoch senior house officer in paediatrics, Birmingham

We welcome articles of up to 600 words on topics such as A memorable patient, A paper that changed my practice, My most unfortunate mistake, or any other piece conveying instruction, pathos, or humour. If possible the article should be supplied on a disk. Permission is needed from the patient or a relative if an identifiable patient is referred to. 